\title{
O Uso de Agentes Conversacionais no Apoio do Ensino de Resolução de Problemas Matemáticos: Uma Revisão Sistemática da Literatura
}

\author{
Arcanjo Miguel Mota Lopes, José Francisco de Magalhães Netto, \\ Dhanielly P. R. de Lima
}
Instituto de Computação (IComp) - Universidade Federal do Amazonas (UFAM)
Av. Gen. Rodrigo Otávio, Coroado - Manaus - AM - Brasil
$\{$ amml, jnetto, dhanielly\} @icomp.ufam.edu.br

\begin{abstract}
This paper presents a Literature Systematic Review (LSR) to identify and characterize the works that utilize Conversational Agents (CA) to assist the people in the mathematics problems resolution. The investigation necessity is justified by mathematics problems complexity and as they can be reduced with the technologies aid. The LSR presented in this paper was realized with the basis in the last 18 years (2001-2018) and were analysis 352 paper. The results helped in the identification of the institutions that work with the theme in question and to characterize the research that uses $C A$ in the mathematics problems resolution.
\end{abstract}

Resumo. Este artigo apresenta uma Revisão Sistemática da Literatura (RSL) para identificar e caracterizar os trabalhos que utilizam Agentes Conversacionais (AC) para auxiliar as pessoas na resolução de problemas matemáticos. Tal necessidade de investigação justifica-se pela complexidade dos problemas matemáticos e em como eles podem ser minimizados com o auxilio das tecnologias. A RSL apresentada neste trabalho foi realizada com base nos últimos 18 anos (2001-2018) no qual foi possivel analisar 352 artigos. Os resultados obtidos auxiliaram na identificação das instituições que trabalham com o tema em questão e a caracterizar as pesquisas que envolvem $A C$ na resolução de problemas matemáticos.

\section{Introdução}

O Agente Conversacional (AC), também denominado de chatterbot ou chatbot, é um sistema de software que imitam a interação com pessoas reais por meio do uso de Linguagem Natural e que vêm sendo estudados por décadas [Radziwill 2017]. Devido a sua dinâmica de interação com as pessoas os ACs estão sendo estudados e incluídos em diversos contextos como, por exemplo, no contexto educacional. Uma vez que podem ser utilizados para ampliar os processos de ensino e aprendizagem, atuando como entidades que simulam uma conversa com o estudante e como instrumento de complementação do aprendizado [Bii 2013].

Neste sentido, várias pesquisas têm sido realizadas a fim de contribuir no estudo dos AC que estão inseridos nos ambientes educacionais como os trabalhos de Nye et al. (2014) e Winker et al. (2018). Esses trabalhos destacam o uso de ACs na educação e o quão esses agentes podem ser motivadores para dar apoio ao aprendizado em algumas áreas de ensino como, por exemplo, para auxiliar no aprendizado de língua estrangeira, 
VII Congresso Brasileiro de Informática na Educação (CBIE 2018)

Anais do XXIX Simpósio Brasileiro de Informática na Educação (SBIE 2018)

ou em Geografia, História, ou em disciplinas mais complexas, como Matemática e Física.

Para esta Revisão Sistemática da Literatura (RSL) investigou-se o uso de AC em Matemática, devido a sua ampla utilização no dia a dia e por ser uma disciplina que apresenta dificuldades para alunos e professores quanto ao processo de ensino e aprendizagem [Seffrin et al. 2010]. Outro fator é que não foi encontrada nenhuma RSL relacionada ao uso de AC para apoiar o aprendizado em resolução de problemas matemáticos. Desta forma, é proposto identificar e caracterizar as dificuldades e os ambientes educacionais matemáticos que utilizam AC como ferramenta de apoio para o aprendizado de Matemática com foco em resolução de problemas.

Para relatar esta pesquisa, o artigo está estruturado da seguinte forma: a seção 2 apresenta a metodologia utilizada, descrevendo o objetivo, as questões de pesquisa, a String de busca e as bibliotecas digitais utilizadas, os critérios de Inclusão e Exclusão e os dados de extração. Na seção 3 são apresentados os resultados e as análises dos artigos que passaram pela filtragem; e a seção 4 traz as considerações finais e trabalhos futuros.

\section{Metodologia da Revisão Sistemática}

Este mapeamento segue o método da RSL proposto por Kitchenham (2007), que descreve os passos necessários para identificar, avaliar e interpretar as pesquisas relacionadas a um tema específico. Para aplicar o método, primeiramente foram definidos o objetivo e as questões de pesquisa, em seguida realizou-se a condução da pesquisa. A seleção dos artigos e a extração das informações são descritas nas subseções a seguir.

\subsection{Objetivo e Questões de Pesquisa}

O objetivo desta RSL é identificar e caracterizar a produção de Sistemas Educacionais de Matemática que utilizam AC para apoiar o estudante no desenvolvimento e aperfeiçoamento das habilidades em resoluções de problemas matemáticos. Para atender o objetivo três Questões de Pesquisa (QP) foram elaboradas, sendo elas:

- QP1: Quais contextos estão sendo aplicados os ACs, incluindo os anos escolares, proposta pedagógica, usuários alvos e os conteúdos da Matemática abordados?

- QP2: Quais as características dos ACs foram desenvolvidas e quais as tecnologias utilizadas?

- QP3: Quais as questões de pesquisas deixadas abertas pelos trabalhos selecionados?

\subsection{Condução da Pesquisa}

Para conduzir a revisão, foram selecionadas as cinco principais bases de dados acadêmicos com base nos estudos qualitativos das bibliotecas digitais apresentados por [Buchinger et al. 2014]. Por isso, foram selecionadas as seguintes bases digitais acadêmicas: ACM Digital Library ${ }^{1}$, IEEE Xplore ${ }^{2}$, SpringerLink ${ }^{3}$, Science Direct ${ }^{4}$ e

\footnotetext{
https://dl.acm.org/

https://ieeexplore.ieee.org/Xplore/home.jsp

https://link.springer.com/
} 
VII Congresso Brasileiro de Informática na Educação (CBIE 2018)

Anais do XXIX Simpósio Brasileiro de Informática na Educação (SBIE 2018)

Scopus ${ }^{5}$. Para realizar a busca fez-se necessário a estruturação da String de busca, que foi desenvolvida por intermédio de uma pesquisa informal da literatura para identificar nas bases de dados abertas as palavras-chaves, e depois identificar os termos utilizados na literatura.

Adaptou-se a string de busca resultante (ver Quadro 1) para cada base de dados selecionada de acordo com suas especificidades. Além disso, o Google Scholar ${ }^{6}$ foi adicionado para detectar pesquisas potencialmente relevantes e que normalmente não são indexados nas bases de dados mais comuns da literatura. Deste último, foram analisados os artigos presentes nas 60 primeiras páginas disponíveis na busca.

Quadro 1 - String de Pesquisa

\begin{tabular}{|c|c|}
\hline Conceitos & Termos Alternativos e Sinônimos \\
\hline $\begin{array}{c}\text { Agente } \\
\text { Conversacional }\end{array}$ & $\begin{array}{c}\text { ("chatbot" OR "chat bot" OR "chatterbot" OR "conversational agent" OR } \\
\text { "conversational companion" OR "dialogue agent" OR "pedagogical agent" OR }\end{array}$ \\
\hline \multicolumn{2}{|c|}{$\begin{array}{c}\text { "virtual companion" OR "virtual conversation") } \\
\text { Matemática }\end{array}$} \\
\hline \multicolumn{2}{|c|}{ ("math" OR "mathematics" OR "mathematical") } \\
\hline Contexto & AND \\
\hline
\end{tabular}

Para projetar uma string de busca adequada, foi aplicado uma ampla definição de AC para capturar na literatura os trabalhos disponíveis nos campos da Matemática, Física, Engenharia e Computação que estejam envolvidos no apoio ao ensino e aprendizagem de resolução dos problemas de Matemática. O escopo da pesquisa foi limitado no contexto de informática/computação voltada para a educação com propósito de evitar que retornassem trabalhos que não estivessem no contexto educacional.

Após a estruturação da String de busca e da escolha das bases digitais, foi realizada a busca dos artigos, no qual obtivemos um resultado total de 352 artigos. É válido salientar que a pesquisa foi delimitada para o período de 2001 a 2018. O resultado é apresentado na Tabela 1.

\section{Tabela 1 - Bases digitais e resultados da $1^{\text {a }}$ pesquisa}

\begin{tabular}{|c|c|}
\hline Base Digital & $\mathbf{N}^{\mathbf{o}}$ de Publicações \\
\hline ACM & 4 \\
\hline IEEE & 8 \\
\hline Scopus & 78 \\
\hline Science Direct & 110 \\
\hline Springer & 144 \\
\hline Google Schoolar & 8 \\
\hline TOTAL & $\mathbf{3 5 2}$ \\
\hline
\end{tabular}

\subsection{Extração dos Dados}

Com o propósito de selecionar os trabalhos relevantes e que atendam as questões de pesquisa desta RSL, os seguintes critérios de inclusão e exclusão foram adotados para selecionar os artigos: CI1: O título, resumo ou palavras-chaves devem mencionar

4 https://www.sciencedirect.com/

5 https://www.scopus.com/

6 https://scholar.google.com.br/ 
VII Congresso Brasileiro de Informática na Educação (CBIE 2018)

Anais do XXIX Simpósio Brasileiro de Informática na Educação (SBIE 2018)

explicitamente alguns dos termos contidos na string de busca; CI2: Os estudos devem estar relacionados à Ciência da Computação, Matemática e Engenharia, porém, voltados para resolução de problemas de Matemática; CI3: O artigo deve ser publicado não antes de 2001. Os critérios de exclusão que foram usados neste estudo são descritos a seguir: CE1: Trabalhos em progresso; CE2: Documentos cujo texto integral não está disponível para livre acesso; CE3: Estudos que não estão no contexto educacional; CE4: Estudos secundários, short paper e capítulos de livros.

Em uma análise inicial, ainda na página da máquina de busca, foi realizado o primeiro estudo, onde foram considerados os "Dados básicos" e em uma segunda análise foram extraídos os dados que contribuem para as respostas das questões de pesquisa, sendo esses considerados "Dados QPs", conforme apresento Quadro 2 a seguir.

\section{Quadro 2 - Informações de Extração}

\begin{tabular}{|l|l|}
\hline \multicolumn{1}{|c|}{ Dados Básicos } & \multicolumn{1}{c|}{ Dados QPs } \\
\hline E1. Título do documento & E4: Grade (Ano) escolar em que foi aplicado o estudo \\
\hline E2. Nome(s) do(s) autor(es) & E5: Tecnologias utilizadas para a elaboração do software \\
\hline E3. Ano da publicação do trabalho & E6: Qual temática da disciplina de Matemática foi aplicada \\
\hline E4. Instituição & E7: As contribuições fomentadas pelo trabalho \\
\hline
\end{tabular}

\section{Resultados e Análise}

A presente RSL ocorreu no período de novembro de 2017 a maio de 2018 e os trabalhos selecionados foram analisados de acordo com os critérios de Inclusão e Exclusão apresentados anteriormente. A lista completa dos artigos selecionados e as principais extrações podem ser conferidas no apêndice deste artigo.

\subsection{Overview}

O processo de seleção foi realizado em três etapas. 1) avaliação do título e resumo, procurando artigos que descrevem AC envolvidos na educação. Nesta etapa, os trabalhos duplicados, estudos secundários e shortpaper foram removidos; 2) os artigos foram lidos integralmente e avaliados conforme os critérios de inclusão e exclusão. 3) os 31 artigos resultantes nesta etapa foram lidos novamente, aplicando o critério de exclusão: CE - artigos que relatam o mesmo estudo, sem avaliações, e resultados diferentes. Nesta etapa, a maioria dos estudos foram excluídos por não demonstrar um AC que estivesse envolvido com a resolução de problemas de Matemática, e não apresentar um chat por texto ou "voz" bidirecional com o estudante. Por fim, 11 artigos atenderam os critérios propostos neste trabalho, conforme apresenta a Quadro 3.

Quadro 3 - Overview dos artigos selecionados

\begin{tabular}{|c|c|c|c|c|}
\hline ID & Referências & Instituição & País & Ano \\
\hline 1 & [Nye et al. 2018] & University of Southern California & \multirow{2}{*}{ EUA } & 2018 \\
\hline 2 & [Song 2017] & Sam Houston State University & & 2017 \\
\hline 3 & [Aguiar, Tarouco \& Reategui 2014] & IFF, UFRGS & Brasil & 2014 \\
\hline 4 & [Duangnamol et al. 2013] & NECTEC, JAISC & Tailândia, Japão & 2013 \\
\hline 5 & [Andallaza et al. 2012] & Ateneo de Manila University & Filipinas & \multirow{2}{*}{2012} \\
\hline 6 & [Tamayo \& Pérez-Marín 2012] & ETSII & Espanha & \\
\hline 7 & [Silvervarg 2010] & Linköping University & Suécia & 2010 \\
\hline 8 & [Kumar et al. 2007] & Carnegie Mellon University & \multirow{3}{*}{ EUA } & 2007 \\
\hline 9 & [Razzaq; Hefferman 2004] & Worcester Polytechnic Intitute & & 2004 \\
\hline 10 & [Heffernan et al. 2002] & Universidade de Carnegie Mellon & & \multirow{2}{*}{2002} \\
\hline 11 & [Rasseneur et al. 2002] & LIUM, IUFM, DIDIREM, & França & \\
\hline
\end{tabular}


VII Congresso Brasileiro de Informática na Educação (CBIE 2018)

Anais do XXIX Simpósio Brasileiro de Informática na Educação (SBIE 2018)

\subsection{Respostas para as Questões de Pesquisa}

Os 11 artigos selecionados passaram por um processo de leitura e extração de informações a fim de responder as questões de pesquisas que são descritas a seguir.

- QP1 - Quais contextos estão sendo aplicados os AC, incluindo os anos escolares, proposta pedagógica, usuários alvos e os conteúdos da matemática abordados?

A QP1 nos auxilia a entender em quais níveis de escolaridade estão sendo estudados e os contextos acadêmicos que foram aplicados os AC. Para responder a QP1, fornecemos um resumo descritivo dos contextos de aprendizagem discutidos nos artigos revisados.

Cenários. As aplicações que foram encontradas nos cenários de aprendizagem formal (aprendizagem intencional e estruturada em uma instituição de ensino e formação) estão nos seguintes níveis de educação: Ensino Fundamental [Heffernan \& Koedinger 2002, Rasseneur et al. 2002, Razzaq \& Hefferman 2004, Kumar et al. 2007, Silvervarg 2010, Tamayo \& Pérez-Marín 2012, Andallaza 2012, Song 2017, Nye et al. 2018]; e para graduação está o trabalho Aguiar et al. (2014). No entanto, no trabalho de Duangnamol et al. (2013) não foram encontradas informações sobre o cenário em que foi realizado a validação do seu trabalho.

Currículos. Os tópicos encontrados, tratam do objetivo de ensino e aprendizagem da proposta de cada um dos artigos. A Trigonometria foi o assunto abordado por Aguiar et al. (2014). Já Rasseneur et al. (2002), Razzaq \& Hefferman (2004), Tamayo \& Pérez-Marín (2012), Duangnamol et al. (2013) e Nye et al. (2018) abordam Problemas Simbolização Algébrica, que é um exercício matemático cuja as informações contextuais significam algo sobre o problema que é representado como texto e não como notação matemática. Geralmente este tipo de problema envolve alguma espécie de narrativa que são também ocasionalmente referidos como problemas de histórias. Além disso, temos Heffernan \& Koedinger (2002) que abordaram fatoração de equação e inequação. Kumar (2007) e Andallaza (2012) abordaram os problemas aritméticos que envolvem Frações. Por último, Silvervarg (2010) e Song (2017) abordam os Problemas de Aritmética.

Proposta Pedagógica. Com relação à proposta pedagógica, extraímos aqueles que foram explicitamente mencionados na descrição das atividades de aprendizagem fornecidas nos artigos. Embora alguns não incluam uma referência específica a uma abordagem pedagógica, mas há uma forte presença pedagógica da aprendizagem colaborativa apoiada por computador e aprendizagem online. Além disso, aferimos as características das propostas pedagógicas descritas nas atividades de aprendizagem. Diante disso, Duangnamol et al. (2013) promove o Pensamento Reflexivo (PR), e segundo o autor, a PR é sinônimo de pensamento crítico, que faz parte do processo de referir-se especificamente aos processos de analisar e fazer julgamento sobre o que acontece durante a resolução dos problemas.

Aguiar et al. (2014) utilizam como suporte para ensino de resolução de problemas a representação do conhecimento dos processos de resolução de problemas matemáticos construídos com base nas experiências dos estudantes superdotados, a fim de fornecer ensino baseado em exemplos. Heffernan \& Koedinger (2002) implementou um modelo de diálogos baseado nas observações em tutores e alunos e com isso, entender o máximo possível o que o aluno diz e fornecer feedback positivo, mesmo 
VII Congresso Brasileiro de Informática na Educação (CBIE 2018)

Anais do XXIX Simpósio Brasileiro de Informática na Educação (SBIE 2018)

quando a resposta geral possa estar incorreta, como forma de motivação. Além disso, temos Andallaza (2012) que também utilizou modelos afetivos para fornecer incentivo e apoio aos estudantes. Outra proposta pedagógica levantada por Tamayo \& PérezMarín 2012, Kumar et al. 2007, Silvervarg 2010, Nye et al. 2018] seria a interação social por meio do diálogo com estudantes como meio para guiá-los na resolução dos problemas. Song (2017) declara que sua abordagem pedagógica é o "aprenda ensinando", onde o aluno assume um papel ativo ao ensinar o agente de computador durante a resolução das atividades de Matemática. Por outro lado, Rasseneur et al. (2002) declara que sua proposta se baseia na competição e cooperação gerada pelo AC como forma de engajamento para o estudante.

\section{- QP2: Quais as características dos ACs foram desenvolvidas e quais as tecnologias utilizadas?}

A QP2 busca responder em quais ambientes educacionais ou tipos de tecnologias os AC foram implementados, seja em STI; Ambientes Virtuais de Aprendizagem (AVA) ou ferramentas de autoria. Para responder à questão de pesquisa QP2 foram analisadas as características dos AC implementados, a arquitetura e tecnologias utilizadas na implementação

Características dos Agentes. Dentre os trabalhos classificados, [Razzaq \& Hefferman 2004, Kumar et al. 2007, Duangnamol et al. 2013] não apresentam nenhuma representação visual do $\mathrm{AC}$, contudo, o destaque fica para sua interface ChatSpace, que conduz um diálogo apoiando os estudantes durante a resolução dos conteúdos de Matemática. Além disso, os estudos de Heffernan \& Koedinger (2002), Tamayo \& Pérez-Marín (2012), Andallaza (2012) e Aguiar et al. (2014) possuem AC sem animação, somente uma figura como uma representação visual de um tutor. Contudo, Tamayo \& Pérez-Marín (2012) explicam que o desenvolvimento da interface foi baseado na metodologia User-Centered Design (UCD), onde o principal objetivo é considerar a opinião do usuário, por isso, foram selecionadas as sugestões fornecidas pelos professores que afirmavam, que o uso de AC com características de animações gráficas poderia distrair os estudantes durante a interação das atividades de matemática. No trabalho de Silvervarg (2010) o AC possui uma representação visual evocando empatia por meio de características faciais, como sorriso, contato visual, atitudes positivas, e um rosto expressivo que mostra emoções e estados como pensar e refletir. Por último, Song (2017) e Nye et al. (2018) utilizaram a textura 3D para criar interfaces realistas com movimentos faciais.

Tecnologias. Pesquisar as tecnologias utilizadas nos trabalhos classificados, com a finalidade de compreender o estado da arte dos softwares que podem ajudar os pesquisadores na criação e desenvolvimento de softwares educacionais mais robustos e inteligente, por isso, a necessidade de destacar na QP2 as tecnologias utilizadas em cada trabalho. No trabalho de Aguiar et al. (2014) é utilizado Artificial Intelligence Markup Language (AIML), uma linguagem baseada em XML desenvolvida para criar diálogos semelhantes à linguagem natural por meio de softwares simulando assim, inteligência humana, e para o funcionamento do AC é utilizado o site Pandorabots ${ }^{7}$. Silvervarg (2010) em sua proposta, utilizou um jogo de Matemática desenvolvido por [Pareto 2004] como base para a construção do seu trabalho. Já o trabalho de Andallaza (2012), utilizou o STI Aplusix para apoiar seu trabalho e Heffernan \& Koedinger (2002)

7 https://home.pandorabots.com/en/ 
VII Congresso Brasileiro de Informática na Educação (CBIE 2018)

Anais do XXIX Simpósio Brasileiro de Informática na Educação (SBIE 2018)

utilizou o AlgebraTutor. Kumar et al. (2007) também utilizou uma ferramenta já construída o Cognitive Tutor Authoring Tools (CTAT) voltado para criação de sistemas tutores flexíveis para a resolução de problemas simples e complexos, capaz de suportar múltiplas estratégias que os alunos podem utilizar para resolver problemas. Nye et al. (2018) combinou o AutoTutor com o sistema de aprendizado adaptativo de Aprendizagem em Espaços de Conhecimento ALEKS. Este sistema híbrido de tutoria que utiliza uma arquitetura orientada a serviço para combinar esses dois sistemas. Outros trabalhos baseados em tecnologias web são os de Rasseneur et al. (2002) e Song (2017). Além disso, Tamayo \& Pérez-Marín (2012) utilizaram as tecnologias web com toolkit OpenNLP ${ }^{8}$ por ser uma ferramenta livre voltada para produção de Linguagem Natural e de fácil integração. Além disso, Razzaq e Hefferman (2004) utilizaram para construir sua proposta o Tutor Development Kit (TDK). Por último, Duangnamol et al. (2013), além de combinar a tecnologia Chatbot, agregaram Ontologias pedagógicas para impulsionar o sistema baseado em multiagentes.

\section{- QP3: Quais as questões de pesquisas deixadas abertas pelos trabalhos selecionados?}

A QP3 nos auxilia na investigação das questões de pesquisas que foram levantadas pelos artigos e que podem conduzir a um aprimoramento dos $\mathrm{AC}$ dentro do contexto de ensino e aprendizagem e, para responder a QP3, foram analisados os trabalhos futuros e seções de questões abertas dos artigos.

Considerando as questões em aberto no campo de $\mathrm{AC}$, alguns dos trabalhos mencionaram a extensão de suas propostas, realizando avaliações com grupos de usuários maiores ou diferentes, mas destaca-se a preocupação na melhoria da interação entre o agente e o estudante com foco em criar um ambiente adaptativo para envolver a atenção dos alunos antes de prosseguir com a tentativa de instrução interativa [Tamayo \& Pérez-Marín 2012, Duangnamol et al. 2013, Aguiar et al. 2014 e Nye et al. 2018]. Outra questão, é a preocupação na personalização da conversação para cada modelo (perfil) dos estudantes, tendo em vista, o AC poder inferir no engajamento do estudante a continuar em uma conversa por um período de tempo maior [Kumar et al. 2007, Silvervarg 2010].

\section{Conclusão e Trabalhos Futuros}

Este trabalho teve como objetivo realizar uma RSL sobre os trabalhos que envolvem os AC na educação matemática. Dos 352 artigos levantados nas bases SCOPUS, IEEE, ACM, Science Direct e Springer Link, 11 artigos foram selecionados com base nos critérios de inclusão e exclusão. A partir destes artigos selecionados, foi possível responder as três questões de pesquisa que guiaram este trabalho. Os resultados das análises nos permitem tirar algumas conclusões sobre o uso AC na Matemática.

Primeiro, percebemos que não há uma nomenclatura padronizada sobre o conceito de AC, Chatbots, Agentes Pedagógicos, Assistentes Virtuais, Agentes Animados entre outros. Às vezes, parecem que são termos idênticos, ou apenas maneiras diferentes de se nomear a mesma coisa; e além disso, muitos dos trabalhos que envolviam os termos AC não apresentaram um diálogo bidirecional (chat ou voz) com os estudantes, apenas mensagens e animações. Além disso, nove dos onze artigos enfocam o Ensino Fundamental, o que destaca a necessidade de estudos em outros

8 https://opennlp.apache.org/ 
VII Congresso Brasileiro de Informática na Educação (CBIE 2018)

Anais do XXIX Simpósio Brasileiro de Informática na Educação (SBIE 2018)

ambientes como no Ensino Médio e Graduação. Vale destacar, também, os ambientes não-formais (extraclasse), que são aqueles em que os alunos podem seguir seus próprios meios de aprendizagem. Por último, questões em aberto pelos trabalhos apontam para uma proposta de adaptação dos sistemas de forma mais interativa e flexível, assim como apontam as pesquisas de Raabe \& Giraffa (2006) e Morais et al. (2017).

Com base nos artigos revisados, a nossa proposta oferece a seguinte lista de verificação no uso de AC em apoio aprimoramento das habilidades resolução de problemas de Matemática: a) fornecer detalhes sobre os cenários de aprendizagem para o qual os ACs foram inseridos; b) fornecer detalhes sobre as tecnologias utilizadas para a construção dos ambientes educacionais; c) fornecer detalhes sobre as propostas pedagógicas que se desenvolveram usando os ACs; d) identificar e denotar as questões abertas deixadas pelos trabalhos, para assim, apontar para construção de novos instrumentos e métodos, se aplicável. Com isso o objetivo desta revisão busca fornecer uma estrutura para futuras direções de pesquisa sobre AC no apoio ao progresso do desenvolvimento do estudante no aprendizado de Matemática.

Contudo, os resultados servirão de base para a implementação de um sistema que atenda às necessidades encontradas nesta RSL, onde se busca a adaptação de conteúdo e conversação para engajamento dos estudantes para apoiar e aprimorar as habilidades no processo de aprendizagem de resolução de problemas de Matemática.

Para pesquisas futuras propomos, com base no recente aumento da popularidade dos bots de mensagens: de interfaces simples e textuais [Klopfenstein et al. 2017], investigar e identificar os AC presentes nas plataformas de mensagens on-line, como por exemplo: Facebook, Telegram, Twitter e Slack que estão sendo desenvolvidos para auxiliar na aprendizagem de Matemática, assim como, os AC aplicados ao ensino de ciências exatas em geral.

\section{Agradecimentos}

Agradecemos o apoio financeiro da Fundação de Amparo à Pesquisa do Estado do Amazonas (FAPEAM) através do Edital No 002/2016 e ao Programa de Apoio à PósGraduação stricto sensu - POSGRAD UFAM.

\section{Referências}

Andallaza, T. C. S., Rodrigo, M. M. T., Lagud, M. C. V., Jimenez, R. J. M., \& Sugay, J. O. (2012). Modeling the Affective States of Students Using an Intelligent Tutoring System for Algebra. In Proceedings of The Third International Workshop on Empathic Computing (IWEC 2012) (Vol. 3, p. 4).

Aguiar, E. V. B., Tarouco, L. M. R., \& Reategui, E. (2014). Supporting problem-solving mathematics with a conversational agent capable of representing gifted students'knowledge. In System Sciences (HICSS), 2014 47th Hawaii International Conference on, pages 130-137. IEEE.

Bii, P. (2013). Chatbot technology: A possible means of unlocking student potential to learn how to learn. Educational Research, 4(2):218-221.

Buchinger, D., de Siqueira Cavalcanti, G. A., and da Silva Hounsell, M. (2014). Mecanismos de busca acadêmica: uma análise quantitativa. Revista Brasileira de Computação Aplicada, 6(1):108-120. 
VII Congresso Brasileiro de Informática na Educação (CBIE 2018)

Anais do XXIX Simpósio Brasileiro de Informática na Educação (SBIE 2018)

Duangnamol, T., Suntisrivaraporn, B., Supnithi, T., and Ikeda, M. (2013). Engaging reflective thinking in learning mathematics word problems by using a personal tutoring agent. Disponível em https://saki.siit.tu.ac.th/acis2013/uploads_final/50_f22a4dc319da563c248715c632d691ac/T ama_acis2013_cr.pdf>.

Heffernan, N. T., \& Koedinger, K. R. (2002). An intelligent tutoring system incorporating a model of an experienced human tutor. In International Conference on Intelligent Tutoring Systems (pp. 596-608). Springer, Berlin, Heidelberg.

Kitchenham, B., \& Charters, S. (2007). Guidelines for Performing Systematic Literature Reviews in Software Engineering. Version 2.3. Durham, UK.

Klopfenstein, L. C., Delpriori, S., Malatini, S., and Bogliolo, A. (2017). The rise of bots: A survey of conversational interfaces, patterns, and paradigms. In Proceedings of the 2017 Conference on Designing Interactive Systems, pages 555-565. ACM.

Kumar, R., Gweon, G., Joshi, M., Cui, Y., Nwaigwe, A., and Rosé, C. P. (2007). Evaluating the effect of social conversation on learning, interaction, and perceived interdependence in a collaborative math problem-solving environment. Disponível em $<$ http://gerrystahl.net/vmtwiki/carolyn.pdf $>$.

Morais, F., da Silva, J., Reis, H., Isotani, S., \& Jaques, P. (2017). Computação Afetiva aplicada à Educação: uma revisão sistemática das pesquisas publicadas no Brasil. Simpósio Brasileiro de Informática na Educação-SBIE, v. 28, n. 1, p. 163.

Nye, B. D., Pavlik, P. I., Windsor, A., Olney, A. M., Hajeer, M., \& Hu, X. (2018). SKOPE-IT (Shareable Knowledge Objects as Portable Intelligent Tutors): overlaying natural language tutoring on an adaptive learning system for mathematics. International Journal of STEM Education, 5(1), 12

Nye, B. D., Graesser, A. C., and Hu, X. (2014). Autotutor and family: A review of 17 years of natural language tutoring. International Journal of Artificial Intelligence in Education, 24(4):427-469.

Pareto, L. (2004). The squares family: A game and story based microworld for understanding arithmetic concepts designed to attract girls. In EdMedia: World Conference on Educational Media and Technology, pages 1567-1574. Association for the Advancement of Computing in Education (AACE).

Raabe, A. L. A., \& Giraffa, L. M. M. (2006, November). Uma arquitetura de tutor para promover experiências de aprendizagem mediadas. Simpósio Brasileiro de Informática na Educação-SBIE, v. 1, n. 1, pp. 278-287.

Radziwill, Nicole M \& Benton, M. C. (2017). Evaluating quality of chatbots and intelligent conversational agents. arXiv preprint arXiv:1704.04579.

Rasseneur, D., Delozanne, E., Jacoboni, P., \& Grugeon, B. (2002). Learning with virtual agents: Competition and Cooperation in AMICO. In International Conference on Intelligent Tutoring Systems (pp. 61-70). Springer, Berlin, Heidelberg.

Razzaq, L. M., \& Heffernan, N. T. (2004, August). Tutorial dialog in an equation solving intelligent tutoring system. In International Conference on Intelligent Tutoring Systems (pp. 851-853). Springer, Berlin, Heidelberg.

Seffrin, H., Rubi, G., da Cruz, B., \& Jaques, P. (2010). Resolvendo equações algébricas no STI PAT2Math. Simpósio Brasileiro de Informática na Educação-SBIE, v. 1, n. 1.

Silvervarg, A. (2010). Development methods for a social conversational agent in a virtual learning environment with an educational math game. In EdMedia: World Conference on 
VII Congresso Brasileiro de Informática na Educação (CBIE 2018)

Anais do XXIX Simpósio Brasileiro de Informática na Educação (SBIE 2018)

Educational Media and Technology, pages 1218-1223. Association for the Advancement of Computing in Education (AACE).

Song, D. (2017). Designing a Teachable Agent System for Mathematics Learning. Contemporary Educational Technology, 8(2), 176-190.

Tamayo, S. and Pérez-Marín, D. (2012). An agent proposal for reading understanding: Applied to the resolution of maths problems. In Computers in Education (SIIE), 2012 International Symposium on, pages 1-4. IEEE.

Winkler, Rainer \& Söllner, M. (2018). Unleashing the potential of chatbots in education: A state-of-the-art analysis. Disponível em < https://www.alexandria.unisg.ch/253983/2/Submission_AoM_Rainer\%20Winkler_Matthias $\% 20$ Soellner.pdf $>$.

Apêndice 1. Extração dos Estudos Selecionados

\begin{tabular}{|c|c|c|c|c|c|}
\hline Trabalho & QP1 & E6 & QP2 & $\mathbf{E 5}$ & QP3 \\
\hline [Nye et al. 2018] & $\begin{array}{c}\text { Ensino } \\
\text { Fundamental }\end{array}$ & $\begin{array}{l}\text { Problemas } \\
\text { algébricos }\end{array}$ & $\begin{array}{l}\text { Textura 3D para } \\
\text { criar faces de } \\
\text { realistas com } \\
\text { movimentos faciais }\end{array}$ & $\begin{array}{l}\text { STI } \\
\text { AutoTutor e } \\
\text { ALEKS tutor }\end{array}$ & $\begin{array}{l}\text { Aprimorar a detecção das reações } \\
\text { dos estudantes identificando tipos de } \\
\text { problemas e outras estratégias } \\
\text { cognitivas }\end{array}$ \\
\hline [Song 2017] & $\begin{array}{c}\text { Ensino } \\
\text { Fundamental }\end{array}$ & $\begin{array}{l}\text { Problemas de } \\
\text { Aritmética }\end{array}$ & $\begin{array}{l}\text { Textura 3D para } \\
\text { criar faces de } \\
\text { realistas com } \\
\text { movimentos faciais }\end{array}$ & $\begin{array}{l}\text { Tecnologias } \\
\text { web }\end{array}$ & $\begin{array}{l}\text { Investigar a contribuição do Agente } \\
\text { na motivação dos alunos, } \\
\text { considerando a diferença individual } \\
\text { em habilidades cognitivas }\end{array}$ \\
\hline $\begin{array}{l}\text { [Aguiar, Tarouco } \\
\text { \& Reategui 2014] }\end{array}$ & Graduação & $\begin{array}{l}\text { Problemas } \\
\text { trigonometria }\end{array}$ & $\begin{array}{l}\text { Representação } \\
\text { visual sem } \\
\text { animações gráficas, } \\
\text { apenas vídeo }\end{array}$ & $\begin{array}{l}\text { Pandorabots e } \\
\text { linguagem } \\
\text { AIML }\end{array}$ & $\begin{array}{l}\text { Tornar o modelo de instrução } \\
\text { interativo }\end{array}$ \\
\hline $\begin{array}{l}\text { [Duangnamol et } \\
\text { al. 2013] }\end{array}$ & N/a & $\begin{array}{l}\text { Problemas de } \\
\text { Simbolização } \\
\text { Algébrica }\end{array}$ & $\begin{array}{l}\text { Sem figura } \\
\text { representação } \\
\text { visual e animações } \\
\text { gráficas }\end{array}$ & $\begin{array}{l}\text { Ontologias e } \\
\text { multiagentes }\end{array}$ & $\begin{array}{l}\text { Tornar o sistema adaptativo as } \\
\text { preferências dos estudantes }\end{array}$ \\
\hline [Andallaza 2012] & $\begin{array}{c}\text { Ensino } \\
\text { Fundamental }\end{array}$ & $\begin{array}{lr}\text { Problemas } & \text { de } \\
\text { fatoração } & \text { de } \\
\text { equação } & \mathrm{e} \\
\text { inequação } & \end{array}$ & $\begin{array}{l}\text { Representação } \\
\text { visual sem } \\
\text { movimentos } \\
\text { gráficos }\end{array}$ & STI Aplusix & $\begin{array}{l}\text { (1) Integração de modelos de } \\
\text { iteração, (2) inclusão de respostas } \\
\text { adicionais, (3) revisões para a } \\
\text { aparência do agente. }\end{array}$ \\
\hline $\begin{array}{l}\text { [Tamayo \& } \\
\text { Pérez-Marín } \\
\text { 2012] }\end{array}$ & $\begin{array}{c}\text { Ensino } \\
\text { Fundamental }\end{array}$ & $\begin{array}{l}\text { Problemas de } \\
\text { Simbolização } \\
\text { Algébrica }\end{array}$ & $\begin{array}{c}\text { Representação } \\
\text { visual sem } \\
\text { animações gráficas }\end{array}$ & $\begin{array}{l}\text { Tecnologia } \\
\text { web e toolkit } \\
\text { OpenNLP }\end{array}$ & Criar modelo de instrução interativo \\
\hline [Silvervarg 2010] & $\begin{array}{c}\text { Ensino } \\
\text { Fundamental }\end{array}$ & $\begin{array}{l}\text { Problemas de } \\
\text { aritmética }\end{array}$ & $\begin{array}{c}\text { Representação } \\
\text { visual com } \\
\text { movimentos faciais }\end{array}$ & $\begin{array}{l}\text { Baseado em } \\
\text { jogo }\end{array}$ & $\begin{array}{l}\text { Personalização da Conversação } \\
\text { conforme preferências do estudante }\end{array}$ \\
\hline $\begin{array}{l}\text { [Kumar et al. } \\
\text { 2007] }\end{array}$ & $\begin{array}{c}\text { Ensino } \\
\text { Fundamental }\end{array}$ & $\begin{array}{l}\text { Problemas } \\
\text { aritméticos de } \\
\text { fração }\end{array}$ & $\begin{array}{c}\text { Representação } \\
\text { visual sem } \\
\text { animações gráficas }\end{array}$ & $\begin{array}{l}\text { Cognitive } \\
\text { Tutor } \\
\text { Authoring } \\
\text { Tools }\end{array}$ & $\begin{array}{l}\text { Personalização da Conversação } \\
\text { conforme preferências do estudante }\end{array}$ \\
\hline $\begin{array}{c}\text { [Razzaq; } \\
\text { Hefferman 2004] }\end{array}$ & $\begin{array}{c}\text { Ensino } \\
\text { Fundamental }\end{array}$ & $\begin{array}{c}\text { Problemas } \\
\text { Álgebra Linear }\end{array}$ & $\begin{array}{l}\text { Sem representação } \\
\text { visual e animação } \\
\text { gráfica }\end{array}$ & \begin{tabular}{|c|} 
Tutor \\
Development \\
Kit
\end{tabular} & $\begin{array}{l}\text { (1) Empregar mais estratégias de } \\
\text { tutoria; (2) Escolha Dinâmica de } \\
\text { Estratégias de ensino }\end{array}$ \\
\hline [Rasseneur 2002] & $\begin{array}{c}\text { Ensino } \\
\text { Fundamental }\end{array}$ & $\begin{array}{l}\text { Problemas } \\
\text { algébricos }\end{array}$ & N/a & JAVA & Melhorias na ferramenta \\
\hline [Heffernan 2002] & $\begin{array}{c}\text { Ensino } \\
\text { Fundamental }\end{array}$ & $\begin{array}{l}\text { Simbolização } \\
\text { Algébrica }\end{array}$ & N/a & $\begin{array}{c}\text { STI } \\
\text { AlgebraTutor }\end{array}$ & $\begin{array}{l}\text { Determinar os ganhos na } \\
\text { aprendizagem ou na motivação. }\end{array}$ \\
\hline
\end{tabular}

